\title{
Analysis on the Organic Photosensitive Diode Structure Optimization based on the Optical System
}

\author{
Yanmin Zhou ${ }^{1, a}$, Jianfang $\mathrm{Li}^{1, \mathrm{~b}}$ \\ ${ }^{1}$ Chongqing College of Electronic Engineering, Chongqing 401331, China \\ a zhouyanmin023@126.com, b lijianfang023023@126.com
}

Keywords: The optical system, Organic photosensitive diode, Structure optimization

\begin{abstract}
Due to the technology and the technology relatively mature, the monocrystalline silicon has been the preferred substrate material in the research of photoelectric device. Compared with the difficulty of traditional inorganic semiconductor materials processing and high production cost, the molecular organic materials and energy level structure can be worked design, fast response, easy processing into a large flexible device, etc. Therefore, the implementation of the function of the organic/inorganic materials compound, and seek complementary functions, in order to obtain a more excellent comprehensive performance of photoelectric functional device, in the field of current research on optoelectronic materials and devices has the attention of people. This paper will discuss the organic photosensitive diode structure optimization based on the optical system.
\end{abstract}

\section{Introduction}

The semiconductor photoelectric detector is the key of the electromechanical integration technology, in the biological, medical, communications, and military and other industries as well as the camera, color copiers, color enlarger photometry and financial business of counterfeit currency identification department has a very wide range of applications, such as is one of the hot spot of research at home and abroad. We study for a big project need produced a new type of silicon photoelectric detector. Difficult to develop this device is active photosensitive area large area and low dark current, and a short wave period of light sensitivity, and has good stability and reliability. With the rapid development of electronic science and technology, composed of photoelectric device equipment, the function of the system is more and more complex; the device number is also increasing, using the environment more and more severe, so the device performance degradation, failure is quite common. The research on the rules of photoelectric device failure, so as to improve the reliability of devices and equipment is an urgent need to solve the problem. But so far the photo sensor, reliability and lifetime of little reported.

\section{The Summary of the Photosensitive Diode}

The photosensitive diode is a kind of light volt device, the types of its general photodiode, avalanche photodiode, blue-ray photosensitive diode, depending on the sensitivity photodiode, infrared photodiode and photosensitive diode laser and ultraviolet photodiode, etc. Mainly used for automatic measurement and control system, as the light FGC circuit, photoelectric sensing device, infrared remote control, infrared security, the street light automatic control, process control, encoder, decoder, etc. In optical measurement technology, optical fiber communication, automatic detection and automatic control technology in the field of application is very extensive. In order to more intuitive understanding of the photosensitive diode structure and principle, analyzes and studies the basic properties of the photodiode, and in the correct use of photovoltaic technology, photosensitive diode characteristics of intelligent test device is designed. 


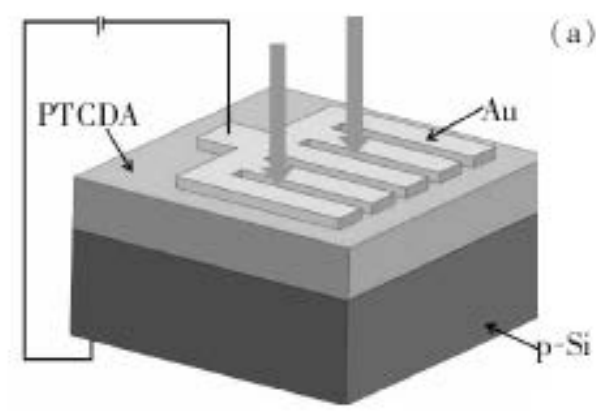

Fig. 1 organic photosensitive diode structure

The photodiode photovoltaic mode suitable for the detection of weak light. Photodiode can work light $\mathrm{v}$ mode (zero bias state), also can work in optical mode (reverse bias) state. The light cases, photodiode current voltage relationship is:

$$
I=I_{0}\left(e^{q v / k T}-1\right)-I_{L}
$$

Among them, $I$ is the output current; $I_{0}$ is for the reverse saturation current; $I_{L}$ is the Photo production current ; $V$ is on the photosensitive diode voltage; $q 、 k 、 T$ is the electronic power, respectively, the boatman constant and thermodynamic temperature. When photosensitive diode work on optical mode, dark current; the photosensitive diode work light volts mode, dark current is zero.

The open circuit voltage of the photosensitive diode law along with the change of light intensity. At the ends of the photosensitive diode under the condition of open circuit, voltage of open circuit voltage $V_{o c}$ :

$$
V_{\text {oc }}=\frac{k T}{q} \ln \left(\frac{q Q A\left(L_{p}+L_{n}\right)}{I_{s}}+1\right)
$$

Among them, $q 、 k 、 T$ is Electronic power, respectively, the bolt Mann constant and the Kelvin temperature; $I_{s}$ is the saturation current; $q Q A\left(L_{p}+L_{n}\right)$ For light current, its size with light intensity changes, according to the type (2) shows that with the increase of light intensity, $V_{o c}$ number increases type in pairs, and under the weak light, $V_{o c}$ is Along with the light intensity change quickly.

\section{The Effect PTCDA Thickness on the Performance of the Device}

Dark current of the photosensitive diode is by reverse saturation current device, barrier area and border of compound of current and surface leakage current. Under reverse bias, the barrier of psi higher level (as shown in figure 2 a dotted line), the built-in potential increase, is advantageous to the hole carrier drift. At this time of dark current is mainly composed of the compound, current drift. But in the absence of light, the number of carrier can move freely in the PTCDA rarely, the drift current is very small. If give light, carrying energy photons into heterojunction, the energy transfer to PTCDA organic layer HOMO energy level in the bound state of electron, make part of the electron transition to LUMO energy levels, thus generated exactions (electron hole pair). If the light raw electronic - hole on is produced in the depletion zone, these electrons - hole to occur in the internal space under the action of electric field drift, drift current. 


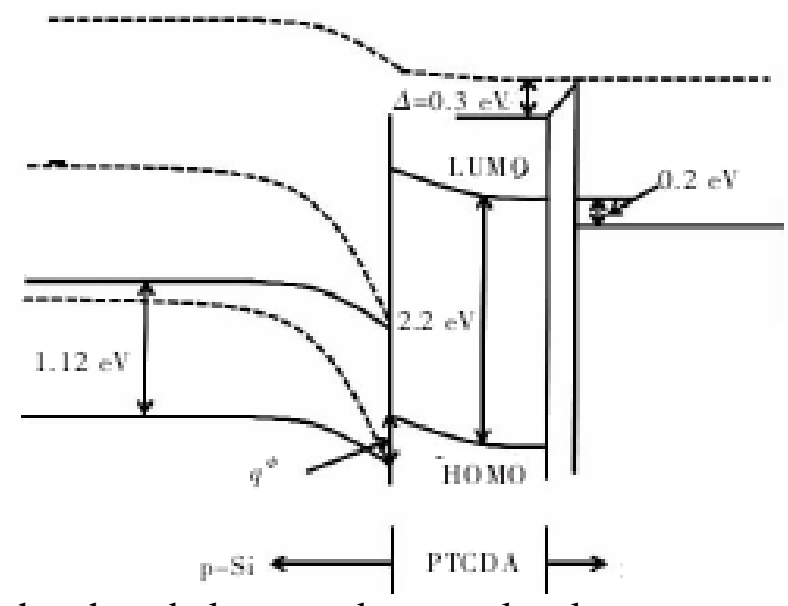

Fig. 2 devices when heat balance and energy level structure under reverse bias

In order to get based on PTCDA organic - inorganic photodiode active layer thickness optimization, this paper studies the PTCDA thickness of $50100150200 \mathrm{~nm}$ respectively the performance of the photodiode. As shown in figure 3 for different thickness of PTCDA membrane absorption in the visible region of the spectrum, the absorption of thin film optical intensity increases with the increase of thickness, and the height of the maximum absorption peak is linear rise with the increase of the thin film thickness (figure 3). Dark current main device produced by internal carrier recombination current decisions. Composite current generated mainly depends on the intrinsic carrier concentration in the silicon substrate and heterojunction barrier height. In addition, some process during the manufacture of the device will affect the quality of silicon surface state and film, which will impact on dark current of the device.

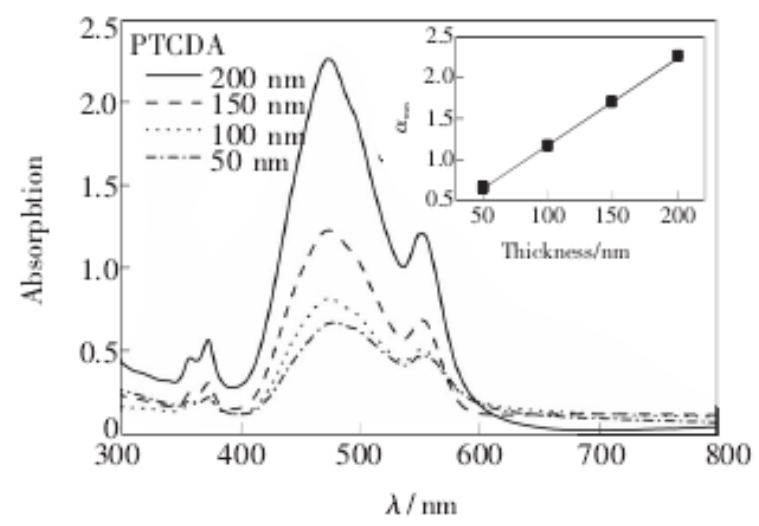

Fig. 3 different maximum absorption peaks of PTCDA film thickness

For photosensitive diode, light current mainly by exhausted area light carrier drift motion formation. By figure 3 is different PTCDA absorption spectrum can be seen that the thickness of the film, the absorption intensity of thin film optical thickness increases, and the number of born and light carrier is with the increase of thickness. Under the same power of light, the light response degree increased with the increase of thickness of PTCDA first then decreases. We speculated that the reasons for this phenomenon mainly by exaction diffusion and transmission in the heterojunction compound, PTCDA exaction diffusion length of about $10.4 \mathrm{~nm}$, therefore with the increase of organic layer thickness, light raw exaction dissociation efficiency drops, causing light to reduce the number of carriers and light current will decrease. On the same device, different power laser irradiation, device light responsively decrease with the increase of light intensity, this phenomenon can be explained by the organic components of the photovoltaic effect. Born in light intensity increases, the light exaction number increasing, while at the same time there is more light raw exaction compound become neutral molecules, thus reducing light exaction dissociation rate. Therefore, with the increase of light intensity, light current eventually reach saturation, in light of current saturated situation, light intensity increases, the degree of light response will decrease. 
Due to restrictions in composite mechanism in charge of non equilibrium zone near the electrodes, and the greater the rate of light $G$ per unit volume, these remaining down carrier will be more. Therefore, under a high $G$ value, photocurrent is likely to turn into space charge limited current. Therefore, we must be clear, whether in a composite model of current limiting began to space charge limited current, we need only observe the current and the relationship between the G. If they are linear relationship, belong to compound limited current, if the relationship is nonlinear, belong to the space charge limited current. Dark current is the main noise sources of the structure of the device, it not only affect the components of the light responsively, but also affect the stability of the device. Therefore, should try to keep the dark current in low value. Theoretical analysis that the main factors of formation of dark current, on the one hand is to form the thermal noise in the heterojunction semiconductor material; On the other hand, the body inside the diode under bias current, current will be increased with the increase of bias.

Due to the small organic molecules/silicon heterojunction photodiode device in the limitation of material and structure, the device to the degree of response of different wavelength of incident light and the response sensitivity is not the same, it will be for a range of wavelengths has the strongest in the spectrum response, and poor response to other light, the light response of the main performance for reverse current and optical response of different. In addition, the spectral response of the device for manufacturing process, the incidence of electrode window material and its thickness and the differences among organic photosensitive layer thickness.

\section{Summary}

Through the research of this paper is systematic review, we can find that there are two shortcomings. First of all, our wide spectral absorption p type organic small molecule/silicon heterojunction photodiode preparation is not enough complete, in the process of the mixture of organic thin film evaporation weight ratio, evaporation temperature of the organic material control, the speed of the rack and the rate of evaporation, etc will affect the quality of the blending system of film, will affect the performance of the device. Although in recent years, there have been many studies reported that the application of the thin films of organic blend system on non-electronic devices, in order to improve the performance of the device. However, for this device physics model of the structure and interface interaction description is not very perfect.

\section{References}

[1] Kang-Jun Baeg, Maddalena Binda, Dario Natali, Mario Caironi, Yong-Young Noh. Organic Light Detectors: Photodiodes and Phototransistors. Adv. Mater. 2013 (31)

[2] Y.Q. Peng, W.L. Lv, B. Yao, G.Y. Fan, D.Q. Chen, P.J. Gao, M.Q. Zhou, Y. Wang. High performance near infrared photosensitive organic field-effect transistors realized by an organic hybrid planar-bulk heterojunction. Organic Electronics. 2013 (4)

[3] SupratimBasak, RajaduraiChandrasekar. Multiluminescent Hybrid Organic/Inorganic Nanotubular Structures: One - Pot Fabrication of Tricolor (Blue-Red-Purple) Luminescent Parallepipedic Organic Superstructure Grafted with Europium Complexes. Adv. Funct. Mater. 2011 (4)

[4] Panagiotis E. Keivanidis, Peter K. H.Ho,Richard H. Friend, Neil C.Greenham. The Dependence of Device Dark Current on the Active - Layer Morphology of Solution - Processed Organic Photodetectors. Adv. Funct. Mater. 2012(22)

[5] Kanwar S.Nalwa,YuankunCai,Aaron L. Thoeming, JosephShinar, RuthShinar, SumitChaudhary. Polythiophene-Fullerene Based Photodetectors: Tuning of Spectral Response and Application in Photoluminescence Based (Bio)Chemical Sensors. Adv. Mater. 2013(37) 